\title{
Analysis of Hepatitis E Virus-Like Sequence in Chimpanzee
}

\author{
Chenglin Zhou ${ }^{1}$; Wang Li ${ }^{1}$; Shixing Yang ${ }^{1, *}$ \\ ${ }^{1}$ Department of Microbiology, School of Medical Science and Laboratory Medicine, Jiangsu University, Zhenjiang, China \\ ${ }^{*}$ Corresponding Author: Shixing Yang, Department of Microbiology, School of Medical Science and Laboratory Medicine, Jiangsu University, Zhenjiang, China. Tel: +86-15006107319, \\ E-mail:yangshixingujs@gmail.com
}

Received: April 14, 2014; Accepted: August 7, 2014

Keywords:Hepatitis E Virus; Chimpanzee; Multilocus Sequence Analysis

\section{Dear Editor,}

Hepatitis E virus (HEV), a member of the genus Hepevirus, is a non-enveloped virus with a positive stranded RNA genome that is approximately $7.2 \mathrm{~kb}$ in length (1). It has been hypothesized that zoonosis is involved in the transmission of $\operatorname{HEV}(2,3)$. Hepatitis E virus antibodies or genes have been reported to exist in many species of mammals, including monkeys $(4,5)$. Recently, divergent HEV strains has been discovered in different animals, including rats (6), mouses (7), and rabbits (8), which suggests that more animal species could be the reservoir of HEV. In the present study, we analyzed a HEV-like sequence, which was found by chance during the discovery of RNA virus in fecal samples of Chimpanzee from a zoo in China. Briefly, we extracted total RNA from the fecal suspension and performed reverse transcription using a primer containing a fixed sequence followed by a randomized octomer at the $3^{-}$end. A single round of DNA synthesis was then performed using Klenow fragment polymerase. Twenty cycles of PCR amplification of nucleic acids was then performed using primers consisting of fixed portions of the random primers. Then the PCR products were purified, cloned into T-vector and sequenced. The resulting sequences were searched in GenBank using BLASTx. Searching results showed that one $685 \mathrm{bp}$ sequence had the highest sequence homology with HEV, sharing $45-58 \%$ sequence identities. Sequence analysis revealed that the putative amino acid sequence of this fragment included the whole RdRp domain, which contained 157 amino acids. Due to the high divergence of the sequence, multiple attempts to acquire longer sequences of this virus failed. In order to investigate whether this sequence is prevalent in the Chimpanzee population, a set of primers were designed according to the $685 \mathrm{bp}$ sequence in the present study to perform PCR screening in 24 fecal samples collected from Chimpanzees at the same zoo and 13 fecal samples from another zoo in China.
The primers were Chev1 [5'-TGTCCTCATGTCTGTCAGG-3'] and Chev2 [5'-AATCACATCTACCAACAGC-3'] for the first round of PCR, and Chev3 [5'-TGCCACGGTCCACCGATCG-3'] and Chev4 [5'-ATAGAACCACCGGCGTTG-3'] for the second round. This set of nested primers was designed to amplify a 154-nt segment. Our PCR screening results indicated that seven $(29.2 \%)$ of the 24 fecal samples from the same zoo were positive for this HEV-like sequence, while none of the 13 samples from the other zoo were positive, which suggests that this virus strain was highly prevalent in the Chimpanzee population at the studied zoo. The seven positive samples were cloned and sequenced; results indicated that they shared $>99 \%$ identity over nucleotide level, suggesting they belonged to the same virus strains. In order to further identify the genetic relationship between the sequences of this study and other known HEV strains, we performed a phylogenetic analysis based on the predicted amino acid sequences in the current study and those related sequences retrieved from GenBank. The HEV sequences included those from well-known HEV genotypes 1-4 (from human or pig), rat HEV, mouse $\mathrm{HEV}$, rabbit HEV, and avian HEV. The other four related virus sequences were also added as outgroups, including hepatitis A virus, sapovirus, rabbit vesivirus and rabbit hemorrhagic disease virus. Briefly, amino acid sequences were aligned using Clusta lW v2.0. Phylogenetic analysis was constructed using the Mega 5 software (http://www. megasoftware.net/). GenBank accession numbers of the sequences used as references in this analysis are shown in Figure 1. The sequences determined in the current study were deposited in GenBank; strain name being NhevCb1 and the accession number KM407530. Our phylogenetic analysis indicated that Nhev-Cb1 clustered with the other HEV sequences, lying in a deep branch with high bootstrap value of 100 (Figure 1). Over the amino acid sequence level, Nhev-Cb1 shared 35-39\% sequence identity 
with the other HEV species, and 29-32\% sequence identities with the four reference sequences. Our phylogenetic results suggested that the virus in the present study was a novel type of HEV. Although the antibodies to HEV and human origin HEV genes were discovered in non-human primates $(4,5,9)$, these animals are not considered as the natural reservoirs for HEV. In the present study, HEV-like sequences were detected in seven (29.2\%) of 24 Chimpanzees at the studied zoo, suggesting that if the sequences are from real viral particles, the virus may be a new type of HEV using non-human primate as its natural host.

Figure 1. Phylogenetic Analysis of the HEV-Like Sequence in the Present Study and the Reference Sequences from GenBank

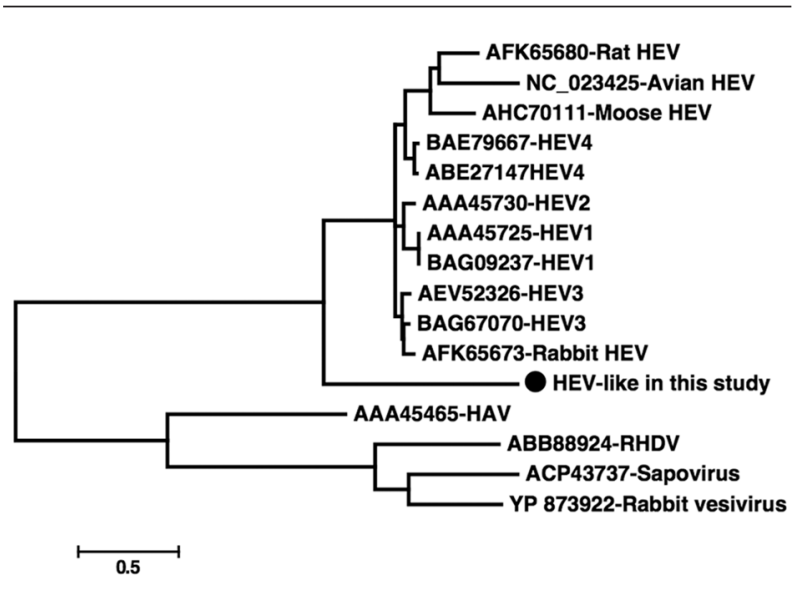

The phylogenetic tree was produced with the amino acid sequence alignments of the sequence in the present study and another 15 reference sequences, using the maximum-likelihood method with Mega 5 software. The sequence identified in the current study is marked with a black circle.

\section{Acknowledgements}

The authors thank the support of the Professional Re- search Foundation for Advanced Talents of Jiangsu University.

\section{Authors' Contributions}

Chenglin Zhou and Shixing Yang conceived the study. Chenglin Zhou and Wang Li performed all the experiments. Chenglin Zhou and Shixing Yang wrote the paper. All authors read and approved the final manuscript.

\section{Funding/Support}

This work was supported by the Professional Research Foundation for Advanced Talents of Jiangsu University under Grant No.12JDG085.

\section{References}

1. Reyes GR, Purdy MA, Kim JP, Luk KC, Young LM, Fry KE, et al. Isolation of a cDNA from the virus responsible for enterically transmitted non-A, non-B hepatitis. Science. 1990;247(4948):1335-9.

2. Meng XJ, Purcell RH, Halbur PG, Lehman JR, Webb DM, Tsareva TS et al. A novel virus in swine is closely related to the human hepatitis E virus. Proc Natl Acad Sci U S A. 1997;94(18):9860-5.

3. Zhang W, He Y, Wang H, Shen Q, Cui L, Wang X, et al. Hepatitis E virus genotype diversity in eastern China. Emerg Infect Dis. 2010;16(10):1630-2.

4. Huang F, Yu W, Hua X, Jing S, Zeng W, He Z. Seroepidemiology and molecular characterization of hepatitis E Virus in Macaca mulatta from a village in Yunnan, China, where infection with this virus is endemic. Hepat Mon. 2011;11(9):745-9.

5. Yamamoto H, Suzuki J, Matsuda A, Ishida T, Ami Y, Suzaki Y, et al Hepatitis E virus outbreak in monkey facility, Japan. Emerg Infect Dis. 2012;18(12):2032-4.

6. Johne R, Heckel G, Plenge-Bonig A, Kindler E, Maresch C, Reetz J, et al. Novel hepatitis E virus genotype in Norway rats, Germany. Emerg Infect Dis. 2010;16(9):1452-5.

7. Lin J, Norder H, Uhlhorn H, Belak S, Widen F. Novel hepatitis E like virus found in Swedish moose. J Gen Virol. 2014;95(Pt 3):557-70.

8. Zhao C, Ma Z, Harrison TJ, Feng R, Zhang C, Qiao Z, et al. A novel genotype of hepatitis $\mathrm{E}$ virus prevalent among farmed rabbits in China. J Med Virol. 2009;81(8):1371-9.

9. Dastgerdi ES, Amini-Bavil-Olyaee S. Hepatitis E virus infection in macaca mulatta. Hepat Mon. 2011;11(10):852-3. 